\title{
Normal stresses in surface shear experiments
}

\section{Journal Article}

Author(s):

Sagis, L. M. C.

Publication date:

2013-05

Permanent link:

https://doi.org/10.3929/ethz-b-000069450

Rights / license:

In Copyright - Non-Commercial Use Permitted

Originally published in:

The European Physical Journal Special Topics 222(1), https://doi.org/10.1140/epjst/e2013-01829-8 


\title{
Normal stresses in surface shear experiments
}

\author{
L.M.C. Sagis ${ }^{1,2, a}$ \\ 1 Food Physics Group, Wageningen University, Bomenweg 2, 6703 HD Wageningen, \\ The Netherlands \\ 2 Polymer Physics, Department of Materials, ETH Zurich, Wolfgang-Pauli-Str. 10, \\ 8093 Zurich, Switzerland
}

Received 16 April 2013 / Received in final form 23 April 2013

Published online 17 June 2013

\begin{abstract}
When viscoelastic bulk phases are sheared, the deformation of the sample induces not only shear stresses, but also normal stresses. This is a well known and well understood effect, that leads to phenomena such as rod climbing, when such phases are stirred with an overhead stirrer, or to die swell in extrusion. Viscoelastic interfaces share many commonalities with viscoelastic bulk phases, with respect to their response to deformations. There is however little experimental evidence that shear deformations of interfaces can induce in-plane normal stresses (not to be confused with stresses normal to the interface). Theoretical models for the stress-deformation behavior of complex fluid-fluid interfaces subjected to shear, predict the existence of in-plane normal stresses. In this paper we suggest methods to confirm the existence of such stresses experimentally.
\end{abstract}

\section{Introduction}

The response of a complex fluid-fluid interface to a deformation is determined by a wide range of parameters. It is well-known that apart from the familiar surface tension, this response is affected by surface rheological properties such as the surface shear and dilatational modulus ([1] and references therein), by the bending rigidity of the interface [1-8], and by its permeability $[8,9]$ (the latter is particularly important for interfaces in vesicles or phase-separated biopolymer systems). In their response to shear deformations viscoelastic interfaces show behavior similar to viscoelastic bulk phases. Their response is characterized by a surface shear storage modulus $G_{s}^{\prime}$, a surface shear loss modulus $G_{s}^{\prime \prime}$, and a set of relaxation times $\tau_{s}$. These properties have been determined experimentally for interfaces, stabilized by a wide range of components, such as polymers, proteins, colloidal particles, and mixtures thereof [1]. It is less well-know if shear deformations of interfaces can also induce normal stresses in the interface, similar to the normal stresses induced in shearing flows of bulk phases. The latter lead to well-known phenomena such as rod-climbing when such bulk phases are stirred with an overhead stirrer, or to die swell in extrusion processes. A study by Olson and Fuller [10] on the behavior of PODMA [poly(octadecyl methacrylate)]

\footnotetext{
${ }^{a}$ e-mail: leonard.sagis@wur.nl
} 

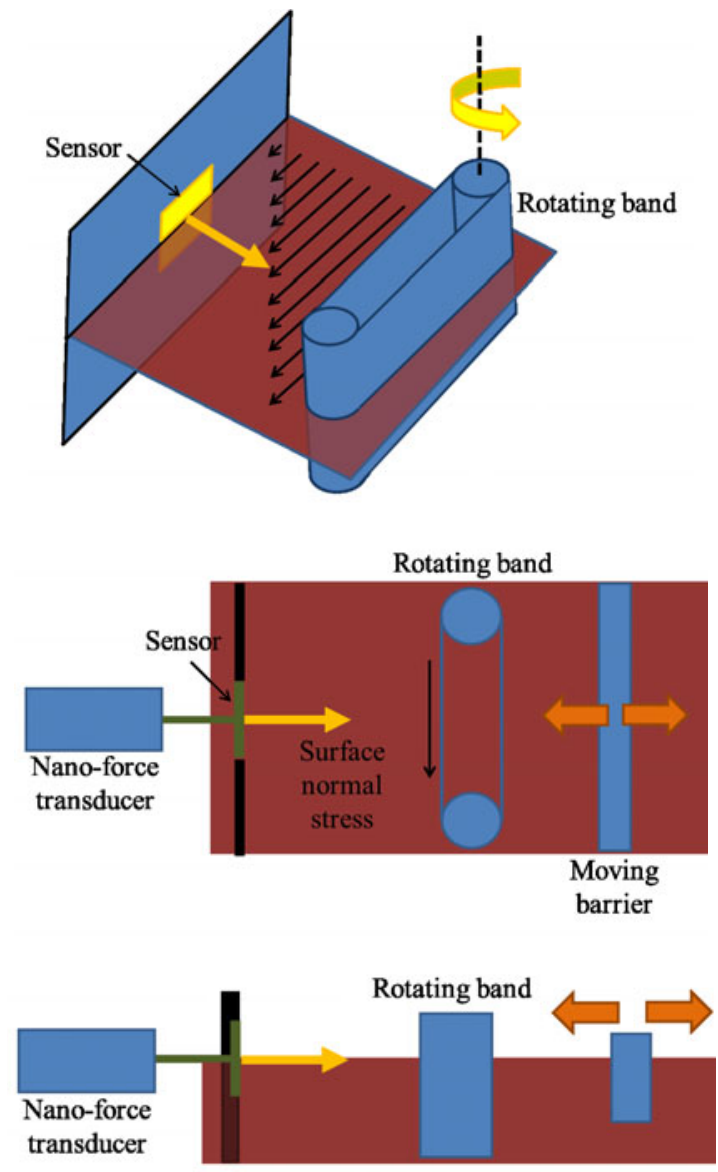

Fig. 1. Three-dimensional view, top view, and side view of a surface shear device for measuring surface normal forces. The surface is sheared using a rotating band. The shear deformation induces a normal force in the direction of the yellow arrow. This force can be measured with a sensor connected to a nano-force transducer. The barrier can be moved to control surface concentration of adsorbed species.

stabilized interfaces in contraction/expansion flows shows evidence of "surface die swell" phenomena, which clearly points to the existence of surface normal stresses.

Surface normal stresses should not be confused with stresses normal to the interface. For a flat interface with surface coordinates $(x, y)$, and $z$ denoting the coordinate perpendicular to the surface, an in-plane shear deformation with magnitude $\gamma_{x y}$ would induce a surface shear stress with magnitude $\sigma_{x y}^{s}$. The surface normal stress (if present) is equal to the stress difference $\sigma_{x x}^{s}-\sigma_{y y}^{s}$, and is a tangential stress difference, oriented perpendicular to the direction of flow (see Figure 1). Stresses normal to the surface would be pointing in the $z$-direction.

When surface normal stresses are induced, they may lead to effects observed also in bulk phases. In surface shear experiments using bi-conical disk [11-15] or doublewall ring geometries [16,17], they could lead to disk- or ring-climbing. Experimental observations of such effects have so far not appeared in the literature (to the knowledge of the author).

There are however theoretical models for the stress-deformation behavior of complex interfaces that predict the existence of such stresses. An example of these is a 
structural model for interfaces stabilized by a mixture of low molecular weight surfactants and rod-like particles, recently developed by Sagis and Öttinger [18,19], in the context of the GENERIC nonequilibrium thermodynamic framework [20-23]. The interface is basically described as a dilute suspension of rod-like particles in a surfactant stabilized interface, and the surface extra stress tensor in this model is given by

$$
\boldsymbol{\sigma}^{s}=\left(\varepsilon_{d}-\varepsilon_{s}\right)\left[\operatorname{tr} \mathbf{D}^{s}\right] \mathbf{P}+2 \varepsilon_{s} \mathbf{D}^{s}+\frac{k_{B} T^{s} \rho_{P}^{s}}{m}\left(\mathbf{C}^{s}-\mathbf{P}\right)
$$

where $\varepsilon_{d}$ and $\varepsilon_{s}$ are the surface dilatational and surface shear viscosity of the part of the interface that is covered by surfactant (described by a linear Bousinesq model), $\mathbf{D}^{s}$ is the surface rate of deformation tensor [15], and $\mathbf{P}$ is the surface projection tensor (the unit tensor for the tangential vector fields) [15]. The last term on the right hand side of (1) describes the contribution of the particles to the surface stress. In this term $k_{B}$ is the Boltzmann constant, $T^{s}$ is the surface temperature, $\rho_{P}^{s}$ is the surface mass density of particles in the interface $\left(\mathrm{kg} / \mathrm{m}^{2}\right)$, and $m$ is the mass of the particles. The second order tensor $\mathbf{C}^{s}$ is the second moment of the particle orientation distribution. It is defined as $\left\langle\mathbf{n}^{s} \mathbf{n}^{s}\right\rangle_{s}$, where $\mathbf{n}^{s}$ is the unit vector denoting the direction of the length axis of the rod-like particles, and the brackets denote a local average. In the model by Sagis and Öttinger $[18,19]$ the time rate of change of this tensor is given by

$$
\begin{aligned}
\frac{\partial \mathbf{C}^{s}}{\partial t}- & \mathbf{C}^{s} \cdot\left(\nabla_{s} \mathbf{v}^{s}\right)^{T}-\left(\nabla_{s} \mathbf{v}^{s}\right) \cdot \mathbf{C}^{s} \\
& +\frac{1}{\tau}\left([1-\beta] \mathbf{C}^{s}-\mathbf{P}+\beta \mathbf{C}^{s} \cdot \mathbf{C}^{s}\right)=0 .
\end{aligned}
$$

Here $\tau$ is a relaxation time, $\beta$ is a parameter describing deviations from linear relaxation behavior, $\nabla_{s}$ is the surface gradient operator [15], and $\mathbf{v}^{s}$ is the surface velocity. The first terms in this expression describe the orientation of the particles in the direction of flow by the imposed velocity gradient, the last terms describe relaxation processes that randomize the orientation by rotational diffusion.

The model described above is able to predict the shear thinning behavior observed experimentally for interfaces stabilized by anisotropic particles [24-26]. An interesting feature of this model is that in steady in-plane shear it predicts the existence of nonzero surface normal stresses. From (1) we find that the surface normal stress difference equals

$$
\sigma_{x x}^{s}-\sigma_{y y}^{s}=\left(\frac{2 k_{B} T^{s} \rho_{P}^{s}}{m}\right)\left(C_{x x}^{s}-1\right)
$$

and this allows us to express the surface normal stress coefficient $\Psi_{s}$ as

$$
\Psi_{s}=\frac{\sigma_{x x}^{s}-\sigma_{y y}^{s}}{\dot{\gamma}^{2}}=\left(\frac{2 k_{B} T^{s} \rho_{P}^{s}}{m}\right) \frac{C_{x x}^{s}-1}{\dot{\gamma}^{2}}
$$

where $\dot{\gamma}$ is the applied surface shear rate. Experimental proof of the actual existence of such stresses would greatly enhance our understanding of the behavior of complex interfaces. The normal stresses predicted by the model described above are quite low. For a dilute $2 \mathrm{D}$ suspension of particles (surface fraction $\sim 0.01$ ), with particle dimensions of the order of $10 \times 1 \times 1 \mu \mathrm{m}^{3}$, a surface density of about $10^{-5} \mathrm{~kg} / \mathrm{m}^{2}$, a particle mass of the order of $10^{-14} \mathrm{~kg}$, and assuming almost full alignment of the particles $\left(C_{x x}^{s} \approx 2\right.$ ), we would obtain (using equation (3)) a normal stress difference of the order $10^{-11} \mathrm{~N} / \mathrm{m}$. Such low stresses would be hard to detect, and are unlikely to have a significant impact on the behavior of an interface during a surface shear experiment. But for interfaces stabilized by a dense layer of particles or polymers these stresses could be several orders of magnitude higher, and might be detectable. In the next section we will suggest a setup that could be used for this purpose. 

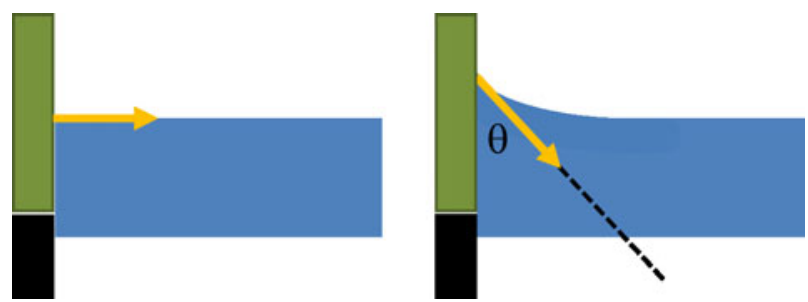

Fig. 2. Effect of contact angle on measured normal force. The measured force equals $F_{n} \sin \theta$, where $\theta$ is the contact angle of the subphase with the sensor.

\section{Suggestions for a device to measure surface normal stresses}

To measure surface normal stresses, we need to subject an interface to a well defined shear deformation. Figure 1 shows a setup based on a Langmuir trough, in which a surface is sheared using a rotating band. A shear field is created between the rotating band and the left (fixed) wall of the trough, and this field induces a normal stress difference at the left wall, pointing in the direction of the rotating band (the yellow arrow in Figure 1). In the left wall a sensor is placed at a vertical level such that the fluid-fluid interface is in contact with the sensor. The sensor could be a highly sensitive pressure sensor, or a rigid plate connected by a rod to a nano-force transducer. The normal force $F_{N}$ measured by the latter would be equal to

$$
F_{N}=\left(\sigma_{x x}^{s}-\sigma_{y y}^{s}\right) L
$$

where $L$ is the width of the sensor plate. High sensitivity force probes (load cells) can measure forces down to a few $\mathrm{nN}$, which would mean that for a sensor width of the order of $0.01 \mathrm{~m}$ the lower limit for the surface normal stress differences we could potentially detect is of the order of $10^{-7} \mathrm{~N} / \mathrm{m}$.

The measured normal force will be affected by the contact angle $\theta$ between the interface and the sensor plate. This is depicted in Figure 2. The force measured by the force transducer will be equal to $F_{N} \sin \theta$, which will be maximal at an angle of $\pi / 2$. So to increase resolution of this method we should choose the material for the sensor plate such that the contact angle is close to $90^{\circ}$. Alternatively, the surface of the sensor plate could be roughened (for example by sand-blasting it).

The setup depicted in Figure 1 is based on a Langmuir trough. The advantage of this setup is the easy control of surface concentration, especially when studying insoluble surface active species, which are spread on the interface. The normal force measurements can also more easily be combined with structural analysis methods for the interface [27]. A drawback of the setup, which uses a rotating band to apply a shear deformation, is that we cannot simultaneously measure the shear stresses. As an alternative, the principle suggested here could be implemented in an oscillating needle rheometer [28], in which the interface is deformed using a thin magnetic needle, displaced by two magnetic coils. The sensor and force transducer should then be placed in the side-wall of the Langmuir trough of this rheometer, parallel to the direction of the needle. A drawback of the needle rheometer is that the range of surface shear rates it can apply is rather limited, and may not be sufficient to produce measurable normal force signals.

In principle the sensor and transducer could also be fitted in the outer wall of a bi-conical disk or double-wall ring geometry, which would also allow simultaneous measurement of surface normal and surface shear stresses. But in these setups it is more difficult to characterize the structure of the interface. Moreover, at high shear rates centrifugal forces may obscure the normal force signals. 


\section{Conclusions and outlook}

In this note we have discussed a possible setup for the measurement of surface normal forces, based on a Langmuir trough, in which a surface shear field is created using a rotating band. The normal forces induced by the shear field can be measured with a sensor located in the fixed wall of the trough, connected to a nano-force transducer. Whether such a setup would be sensitive enough to detect surface normal forces needs to be confirmed by experiments. Confirming the existence of these stresses would greatly enhance our understanding of the dynamics of complex fluid-fluid interfaces.

\section{References}

1. L.M.C. Sagis, Rev. Mod. Phys. 83, 1367 (2011)

2. J.B.A.F. Smeulders, C. Blom, J. Mellema, Phys. Rev. A. 42, 3483 (1990)

3. J.B.A.F. Smeulders, C. Blom, J. Mellema, Phys. Rev. A. 46, 7708 (1992)

4. K.H. de Haas, G.J. Ruiter, J. Mellema, Phys. Rev. E 52, 1891 (1995)

5. E. Scholten, L.M.C. Sagis, E. van der Linden, J. Phys. Chem. B 108, 12164 (2004)

6. E. Scholten, L.M.C. Sagis, E. van der Linden, Macromolecules 38, 3515 (2005)

7. E. Scholten, L.M.C. Sagis, E. van der Linden, J. Phys. Chem. B 110, 3250 (2006)

8. L.M.C. Sagis, J. Controlled Release 131, 5 (2008)

9. E. Scholten, J. Sprakel, L.M.C. Sagis, E. van der Linden, Biomacromolecules 7, 339 (2006)

10. D.J. Olson, G.G. Fuller, J. Non-Newtonian Fluid Mech. 89, 187 (2000)

11. S.G. Oh, J.C. Slattery, J. Colloid Interf. Sci. 67, 516 (1978)

12. D.A. Edwards, H. Brenner, D.T. Wasan, Interfacial Transport Phenomena and Rheology (Butterworth-Henemann, Boston, 1991)

13. P. Erni, P. Fischer, E.J. Windhab, V. Kusnezov, H. Stettin, L. Läuger, Rev. Sci. Instrum. 74, 4916 (2003)

14. P. Erni, P. Fischer, P. Heyer, E.J. Windhab, V. Kusnezov, L. Läuger, Prog. Coll. Polym. Sci. 129, 16 (2004)

15. J.C. Slattery, L.M.C. Sagis, E.S. Oh, Interfacial Transport Phenomena, 2nd edn. (Springer, New York, 2007)

16. O. Regev, S. Vandebril, E. Zussman, C. Clasen, Polymer 51, 2611 (2010)

17. S. Vandebril, A. Franck, G.G. Fuller, P. Moldenaers, J. Vermant, Rheologica Acta 49, $131(2010)$

18. L.M.C. Sagis, H.C. Öttinger (submitted) (2013)

19. L.M.C. Sagis (submitted) (2013)

20. M. Grmela, H.C. Öttinger, Phys. Rev. E. 56, 6620 (1997)

21. H.C. Öttinger, M. Grmela, Phys. Rev. E. 56, 6633 (1997)

22. H.C. Öttinger, Beyond Equilibrium Thermodynamics (Wiley-Intersience, Hoboken, 2005)

23. H.C. Öttinger, D. Bedeaux, D.C. Venerus, Phys. Rev. E 80, 021606 (2009)

24. B. Madivala, J. Fransaer, J. Vermant, Langmuir 25, 2718 (2009)

25. P.F. Noble, O.J. Cayre, R.G. Alargova, O.D. Velev, V. Paunov, J. Am. Chem. Soc. 126, $8092(2004)$

26. M.G. Basavaraj, G.G. Fuller, J. Fransaer, J. Vermant, Langmuir 22, 6605 (2006)

27. E. van der Linden, L.M.C. Sagis, P. Venema, Curr. Opinion Coll. Interf. Sci. 8, 349 (2003)

28. G.F. Brooks, G.G. Fuller, C.W. Frank, C.R. Robertson, Langmuir 15, 2450 (1999) 